\title{
PROPUESTA DE PROTOCOLOS DE MANEJO DE EMERGENCIAS ODONTOLÓGICAS DE LA UNIVERSIDAD CATÓLICA DE CUENCA.
}

\section{PROPOSAL OF DENTAL EMERGENCIES MANAGEMENT PROTOCOLS AT THE CATHOLIC UNIVERSITY OF CUENCA.}

\author{
Sanchez-Zamora Vilmo R. ${ }^{1 *}$ Salgado-Álvarez Rosa E. ${ }^{2}$ Cardenas-Sanchez Elvita P. ${ }^{3}$ Narvaez-Gonzalez Ruth A. ${ }^{4}$ \\ ${ }^{1}$ Médico Docente de la carrera de Odontología de la Universidad Católica de Cuenca. Ecuador. \\ ${ }^{2}$ Odontóloga Rural del Ministerio de Salud Pública del Ecuador. ${ }^{3}$ Egresada de la Universidad Católica de \\ Cuenca.Ecuador.Ecuador. ${ }^{4}$ Egresada de la Universidad Católica de Cuenca.Ecuador.Ecuador. \\ *vsanchezz@ucacue.edu.ec
}

\begin{abstract}
Resumen
OBJETIVO: Proponer un manual de intervención para las emergencias médico- odontológicas más frecuentes en la Clínica Odontológica de la Universidad Católica de Cuenca. MÉTODOS: Se realizó una revisión bibliográfica e investigación de campo, acerca de las emergencias médico-odontológicas que se pueden presentar con mayor frecuencia dentro de la consulta en odontología. Desarrollando esta investigación en la modalidad cualitativa y cuantitativa, ya que se presenta la información mediante un análisis para interpretar datos, haciendo el uso de tablas estadísticas en el análisis de encuestas para la obtención de resultados. RESULTADOS: De las emergencias presentadas en la consulta odontológica, la más frecuente, es la Lipotimia con un $53 \%$; seguida del shock Hipoglicèmico $25 \%$; la crisis hipertensiva y las crisis inducidas con anestésicos locales con un $8 \%$.
\end{abstract}

Palabras clave: Emergencias, consulta, Odontología.

\begin{abstract}
AIM: To propose the protocol of intervention for the most frequent medical-dental emergencies of the dentistry clinic of the Catholic University of Cuenca. MATERIALS AND METHODS: We carried out a literatura review and field research, of which are the most frequent dental doctor emergencies that can be presented within the dental clinic, developing this research in the qualitative and cuantitative modality, since the information is presented through the analysis and interpretation of de data and also makes use of statistical tables in the analysis of surveys to obtain the results and to be able to do the writing. RESULTS: Of the emergencies presented at the dental clinic, the most frequent is Lipotym 53\%; followed by hypoglycemic shock with 25\%; and lastly among the less frequent are anaphylactic shock, hipertemsive crisis and crises induced whit local anesthetics with $8 \%$.
\end{abstract}

Key words: Emergencies,consultation, Dentistry.

\section{INTRODUCCIÓN}

El protocolo de manejo de emergencias es un documento en el que se describe una secuencia sobre el procedimiento de cómo se atiende al paciente en una enfermedad y estado de salud, ayudando así en el diagnóstico y efectividad del tratamiento. Es un instrumento que mejora la calidad de atención en salud, ya que se unen conceptos médicos, o variaciones de conductas entre clínicos, si se promueve un sistema de atención, se disminuyen gastos. Se establecen prácticas confiables para los pacientes, favorecen el tiempo de trabajo y toma de decisiones.
Se puede prevenir una gran cantidad de urgencias y emergencias médicas, conociendo el estado de salud de un paciente. Esto se puede llegar a conocer al momento de realizar la historia clínica, de aquí la importancia de dar una evaluación minuciosa a cada uno de los pacientes. La historia clínica básica, puede alertar sobre posibles problemas médicos, ya que incluyen datos como: antecedentes de enfermedades cardiacas, asma, convulsiones, ingesta de medicamentos, alergias experiencias previas con otros Odontólogos, y la predisposición a alguna enfermedad con los antecedentes familiares. 
Villatoro A. en su revisión bibliográfica de definiciones básicas en medicina de urgencia, determina que urgencia se refiere una condición que requiere atención inmediata y que, sin tratamiento en su debido tiempo puede causar daño prolongado de una o más funciones corporales. Ejemplo: sospecha de fractura. Por otro lado, este autor define a la emergencia como una situación de peligro o desastre que requiere una acción inmediata y que, sin tratamiento el paciente puede sufrir daño severo. Ejemplo herida por arma de fuego. ${ }^{1}$

Santos M. y colaboradores en una revisión bibliográfica sobre urgencias clínico estomatológicas concluyen que debido a que el paciente que es sometido a algún tipo de cirugía estomatológica sea esta mayor o sencilla está predispuesto a sufrir complicaciones durante el proceso debido a los medicamentos administrados para lograr una anestesia los mismos que pueden ocurrir en un abordaje quirúrgico no estomatológico, se refieren a complicaciones en anestesia, complicaciones cardiorrespiratorias, reacciones toxicas y alérgicas entre otras, por lo que el estomatólogo debe estar familiarizado con el manejo de estas complicaciones siendo necesario la creación de protocolos de manejos de urgencias. ${ }^{2}$ Gonzáles I. en su revisión bibliográfica sobre urgencias y emergencias en el gabinete dental realizado en Sevilla en el año 2016, analizó 18 artículos relacionados y concluyó que alrededor del $75 \%$ de los casos los estomatólogos aseguran haber tenido al menos una emergencia o urgencia durante el 2016. Además, este autor señaló que un estudio realizado en Ohio realizado por Kandray DP y colaboradores, un $10.7 \%$ de los casos los dentistas tienen que llamar a una ambulancia o servicio de emergencias debido a una urgencia o emergencia de origen cardiovascular. Por otro lado, Gonzáles luego de su revisión de los 18 artículos determinó que la patología más frecuente fue el sincope vasovagal. Las reacciones alérgicas se dan en un $18,7 \%$ de los casos, las crisis hipertensivas se dan en un $9,5 \%$, las crisis convulsivas en un $16,1 \%$, hipoglucemias y complicaciones de anestesia en un $14,06 \%$, ataques de asma en un $5 \%$, la ansiedad en un $75 \%$, reacciones anafilácticas $3,8 \%$ y con un menor porcentaje el infarto agudo de miocardio con un $1,1 \%$. Gonzales señala que un $66 \%$ de dentistas llegan a un diagnóstico correcto en una situación de emergencia y que un $98 \%$ sabe tomar la tensión arterial, $97 \%$ el pulso carotideo, $62 \%$ son capaces de realizar RCP, $57 \%$ sabe utilizar el Ambu y colocar oxígeno, $32 \%$ establecen una via de acceso venoso, el $16 \%$ conoce como colocar una cánula tipo Guedel, y solo un $3 \%$ analiza un electrocardiograma. ${ }^{3,4}$

De esta manera se puede evidenciar que en las emergencias y urgencias leves la mayoría de los estomatólogos son capaces de llevar un correcto diagnóstico y manejo inicial, mientras que en las graves existe inconsistencia durante el manejo, por lo que es importante diseñar protocolos de manejo inicial que puedan orientar al profesional a realizar un correcto manejo sin poner en riesgo la vida del paciente. . $^{5,6}$ Se propone un manual de intervención para las emergencias médico- odontológicas más frecuentes en la Clínica Odontológica de la Universidad Católica de Cuenca

\section{MATERIALES Y MÉTODOS}

Se realizó una revisión bibliográfica e investigación de campo, acerca de que emergencias médico odontológicas se realizan de manera más frecuente en la clínica de la carrera de Odontología de la Universidad Católica de Cuenca, en este estudio se realizó una encuesta dirigida a profesionales en el campo odontológico, que alguna vez presentaron un tipo de emergencia en la clínica de la Universidad, tomando en cuenta a los pacientes de los estudiante de octavo, noveno y décimo ciclo respectivamente que acudieron a la Clìnica, para hacer un análisis de su estado general de salud y poder recibir el tratamiento odontológico adecuado. En este estudio se incluyó datos de pacientes que presentaron algún tipo de emergencia al momento del tratamiento odontológico, y se excluyó a los pacientes que no presentaron ninguna emergencia y tuvieron un curso normal. El tamaño de muestra aplicada a este estudio fue por conveniencia.

\section{EMERGENCIA MÉDICA EN CONSULTA ODONTOLÓGICA}

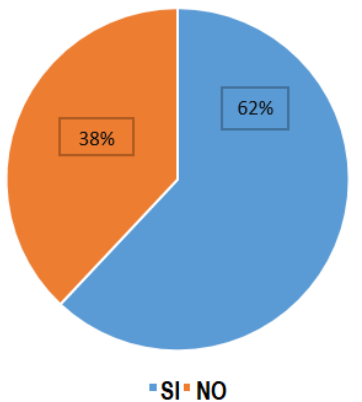

Fig. 1. PREVALENCIA DE EMERGENCIAS MÉDICO ODONTOLÓGICAS EN LA CLÍNICA ODONTOLÓGICA.

\section{EMERGENCIAS EN LA CONSULTA ODONTOLÓGICA}

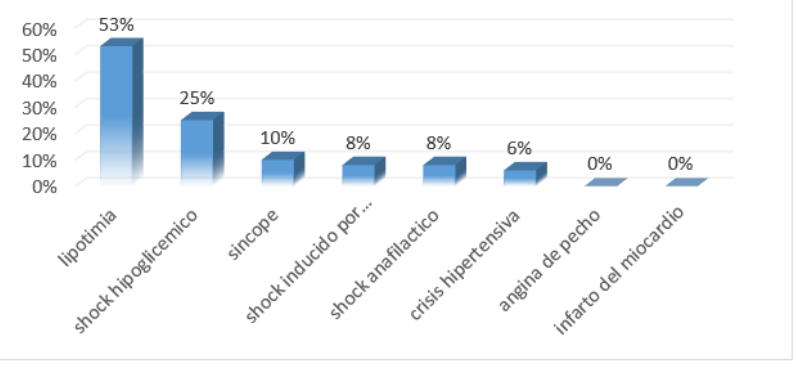

Fig. 2. EMERGENCIAS MÁS FRECUENTES EN LA CONSULTA ODONTOLÓGICA 


\section{RESULTADOS}

De los profesionales en Odontología encuestados, existió mayor prevalencia de Profesionales Odontólogos, que si atendieron emergencias médico odontológicas durante un procedimiento, teniendo un menor resultado aquellos profesionales que no se les presento algún tipo de emergencia durante el tratamiento odontológico. (Gráfico 1) De las emergencias presentadas en la consulta odontológica, se dividió en dos grupos las más frecuentes siendo, la lipotimia con un mayor resultado, seguida del shock hipoglucémico y dentro de las menos frecuentes se encuentran el síncope, shock inducido por anestésicos, shock anafiláctico, la crisis hipertensiva. (Gráfico 2).

\section{PROPUESTA DE LAS DIFERENTES EMERGEN- CIAS MÉDICO ODONTOLÓGICAS}

Se presenta la siguiente propuesta como resultado de la revisión de la literatura especializada.

\begin{tabular}{|c|c|}
\hline EMERGENCIA & PROTOCOLO \\
\hline & 1. SUSPENDER FARMACOS \\
\hline & 2. SUSPENDER ANESTESIA \\
\hline & 3. ASEGURAR FUNCIÚN \\
\hline & RESPIRATORIA \\
\hline SHOCK ANAFILÁCTICO Y & 4. POSICIÓN TREN DE LEMBURG \\
\hline \multirow[t]{6}{*}{ ANESTÉSICOS LOCALES } & 5. FORMA SSEVERA: \\
\hline & ADMINISTRAR ADRENALINA \\
\hline & 6. REACCIONES TARDIAS: \\
\hline & ADMINISTRAR CORTICOIDES \\
\hline & 7. CHOQUE SEVERO \\
\hline & PROLONGADO:UCI \\
\hline
\end{tabular}

Tabla 1. Protocólo de atención frente a un Shock Anafiláctico y un Shock por anestésicos locales.

\begin{tabular}{|l|l|}
\hline EMERGENCIA & PROTOCOLO \\
\hline \multirow{4}{*}{ ANGINA DE PECHO } & 1. SUSPENDER EL TX DENTAL \\
& 2. COLOCAR EN POSICIÓN A 45 \\
& 3. EPISODIO AGUDO: \\
& ADMINISTRAR \\
& NITROGLICERINA SUB. \\
& 4. EPISODIO PROLONGADO: \\
DINITRATO DE ISOSORBIDE \\
C/5M \\
\\
5. TOMAR SIGNOS VITALES Y \\
ADMINISTRAR OXIGENO \\
\hline
\end{tabular}

Tabla 2. Protocólo de atención frente a una Angina de Pecho.

\section{DISCUSIÓN}

En el campo de la Salud, sobre todo en Odontología el bienestar del paciente que acude es incierta, por el motivo que al realizarle preguntas dentro de la anamnesis no siempre manifiestan la verdad por diversas circunstancias, por vergüenza, por timidez o por no conocer alguna enfermedad que

\begin{tabular}{|l|l|}
\hline EMERGENCIA & PROTOCOLO \\
\hline \multirow{4}{*}{ INFARTO AL MIOCARDIO } & 1. OXIGENO \\
& 2. ANALGÉSICOS: DOLOR \\
& INSOPORTABLE MORFINA \\
& 3. NITRATOS \\
4. DISMINUIR LA TENSIÓN & ARTERIAL: \\
& BETABLOQUEANTES \\
& 5. ADMINISTRAR CALCIO \\
& ANTAGONISTASO \\
& BLOQUEADORES DE LOS \\
& CANALES DE CALCIO \\
\hline
\end{tabular}

Tabla 3. Protocólo de atención frente a un infarto del miocardio.

\begin{tabular}{|c|c|}
\hline EMERGENCIA & PROTOCOLO \\
\hline SHOCK HIPERTENSIVO & $\begin{array}{l}\text { 1. } \\
\text { EVITAR CAUSA DE ESTRÉS } \\
\text { 2. EVITAR ESTIMULOS } \\
\text { DOLOROSOS } \\
\text { 3. } \text { CONTROL DE PRESIÓN } \\
\text { ARTERIAL } \\
\text { 4. POSICIÓN DE TREN DE } \\
\text { LEMBURGO } \\
\text { 5. ADMINISTRAR NIFEDIPINO } \\
\text { SUBLINGUAL } \\
\text { 6. EN CASO DE FRACASODE } \\
\text { NIFEDIPINO CAPTOPRIL. } \\
\text { 7. FRENTE AL FRACASO DE LAS } \\
\text { 2 CLONIDINA }\end{array}$ \\
\hline
\end{tabular}

Tabla 4. Protocólo de atención frente a un Shock Hipertensivo.

padezcan. Con este antecedente se procedió a realizar una búsqueda de alternativas, de los diferentes acontecimientos inesperados que se presentan en la Consulta Odontológica. ${ }^{4}$ Como primera instancia se tiene, que según los resultados obtenidos por encuestas realizadas a los Odontólogos en el $62 \%$ respondieron afirmativamente a tener una emergencia médico odontológica mientras que el $38 \%$ tuvo una respuesta negativa a tener una emergencia. Se asemeja con el estudio de Paredes J, en el año 2011 con el artículo de "Protocolos para emergencias médico odontológicas más frecuentes en la zona centro del País." ${ }^{7}$ En el cual asegura que existe un $67.5 \%$ que presentaron una emergencia médico odontológica mientras que existe un $32,5 \%$ que no presentaron una emergencia médico-odontológica. Sin embargo difiere con el artículo de Albornoz L. en el año 2016 con el artículo de "Emergencias médicas más frecuentes ocasionadas durante el tratamiento odontológico en pacientes atendidos en el Hospital Hermilio Valdizán Huánuco, 2016". ${ }^{8}$ En el que asegura que tiene un $97,3 \%$ que no presentaron emergencias médicas y un 2,7 si presentaron emergencias médicas odontológicas.

En el resultado obtenido en emergencias presentadas en la consulta Odontológica en la Universidad Católica de Cuenca fue la Lipotimia con $53 \%$ corrido de Shock Hipoglucémico $25 \%$. Otros con $17 \%$ y Crisis Hipertensiva 


\begin{tabular}{|c|c|}
\hline EMERGENCIA & PROTOCOLO \\
\hline \multirow{13}{*}{ SHOCK HIPOGLUCÉMICO } & 1. MONITOREAR LOS NIVELES \\
\hline & DE GLUCOSA \\
\hline & 2. SUSPENDER EL TX DENTAL \\
\hline & 3. ADMINISTRAR 15GR DE \\
\hline & CARBOHIDRATOS CADA 15 \\
\hline & MINUTOS HASTA LLEGAR A \\
\hline & LOS NIVELES NORMALES. \\
\hline & 4. REPETIR POR 3 VECES \\
\hline & 5. SI LOS NIVELES NO LLEGAN A \\
\hline & LA NORMALIDAD \\
\hline & 6. ADMINISTRAR POR IV \\
\hline & SOLUCIÓN DE DEXTROSA O \\
\hline & GLUCAGON. \\
\hline
\end{tabular}

Tabla 5. Protocólo de atención frente a un Shock Hipoglucémico.

\begin{tabular}{|l|l|}
\hline EMERGENCIA & PROTOCOLO \\
\hline \multirow{5}{*}{ SÍNCOPE } & 1. INTERRUMPIR LA ATENCIÓN. \\
& 2. SACAR TODO MATERIAL DE \\
& LA BOCA \\
& 3. HABLAR ACTIVAMENTE CON \\
& EL PCTE \\
& 4. POSICIÓN SUPINA \\
5. PROPORCIONAR AIRE \\
6.VIGILAR SI EL PCTE RESPIRA \\
7. CON SIGNOS VITALES ENVIAR \\
A CASA. \\
\hline
\end{tabular}

Tabla 6. Protocólo de atención frente a un Síncope.

$8 \%$, Shock Anafiláctico y Crisis inducida por anestésicos locales. La Crisis Epilépticas, Angina de pecho e Infarto al miocardio con $0 \%$ de la investigación. Difiere con el estudio de Gutiérrez P, Rivera G en el año 2012 con el artículo de "Importancia actual de las urgencias médicas en el consultorio dental" el cual asegura que el Sincope es la primera emergencia odontológica con un 50,32\%, seguido con una reacción alérgica moderada con un $8,43 \%$, angina de pecho con un $8,33 \%$, un $8,08 \%$ de hipotensión postural, un $5,21 \%$ de convulsiones, $4,54 \%$ con ataque de asma, un $4,33 \%$ con hiperventilación, $2,0 \%$ en hipoglucemia, $0,99 \%$, $0,66 \%$ con una sobredosis de anestésicos locales. También difiere con el estudio de Paredes J. en el año 2011 con el artículo de "Protocolos para emergencias médico odontológicas más frecuentes en la zona central del País". ${ }^{9}$ En el cual asegura que existe en primer lugar,la crisis hipertensiva con $90 \%$, seguida de pacientes diabéticos no controlados con $80 \%, 42 \%$ otro tipos de emergencias, $40 \%$ para pacientes que poseen alergia a anestésicos locales, $35 \%$ crisis epilépticas, $11 \%$ para pacientes que presentan una emergencia con MIC, $10 \%$ para pacientes con alguna reacción anafiláctica, $9 \%$ para pacientes con Sincope y un 5\% para pacientes con angina de pecho. Difiere con el estudio de Albornoz L en el año $2016 .{ }^{10}$ en el cual asegura que existe como primera emergencia médica odontológica la crisis hipertensiva con un $1,3 \%$, seguido del sincope con un $0,7 \%$ y la hiperglucemia con un $0,7 \%$.

\section{CONCLUSIÓN}

- Se determinó que existe un $62 \%$ de odontológos que han atendido alguna emergencia médico odontológica.

- La emergencias que ocurren de manera más frecuente fue Lipotimia (Desmayos) con un 53\% seguido de Shock Hipo glucémico $25 \%$. Otros con $17 \%$ y Crisis Hipertensiva $8 \%$, Shock Anafiláctico y Crisis inducida por anestésicos locales. La Crisis Epilépticas, Angina de pecho e infarto al miocardio con $0 \%$ de la investigación.

- Sé presentó una propuesta de protocolo de atención de emergencias médicas en el consultorio dental.

\section{Referencias}

1 Martínez Villatoro A. Definiciones básicas en Medicina de Urgencia. México DF. 2005.

2 Aldereguía Lima G. Urgencia clínico-estomatológicas. Guías para el diagnostico y tratamientos. Rev. Cubana de Estomatología 2000;37 (1); 5-49.

3 Rovira F. Concepto de urgencias. Aspectos éticos-legales. En: Rovira E, editor. Urgencia en Enfermeria. Madrid: Difusión avances de Enfermeria; 2005.p17-29.

4 Paredes Jenny. "Protocolos para emergencia medico odontológicas mas frecuentes en la zona centro del país". UNIANDES. 2011-

5 Sagrista J. Abordaje, diagnóstico y Terapéutico de Sincope en Urgencias. Emergencias 2017;19: 273-82.

6 Gonzalez I. Técnicas y procedimientos de Enfermeria. Madrid: Difusión avances 2009.

7 Martinez Sahuquillo A. Prevención y manejo de las situaciones de emergencia en el gabinete odontológico. En: Machuca G, Bullón P editores. Tratamiento odontológico en pacientes especiales. Madrid: Laboratorios Normon 3013. P.79-108.

8 Salcedo R. (online); 2008 (cited 2016 Noviembre 20. Disponible en: www.geocities.com/amirhali/fpcass/protocolosmedicos.

9 Lalama M. "Buenas prácticas de prescripción. Los 100 protocolos terapéuticos".;2008.

10 Salcedo R. (online); 2008 (cited 2016 Noviembre 20. Disponible en: www.geocities.com/amirhali/fpcass/protocolosmedicos.

Recibido: 12 de Junio de 2018.

Aceptado: 26 de Julio de 2018. 